\title{
Integration of Requirements for Performance Indicator System Definition and Implementation Methods
}

\author{
Michel Ravelomanantsoa, Yves Ducq, and Bruno Vallespir \\ University of Bordeaux, IMS-LAPS/GRAI, UMR 5218 CNRS \\ 351 Cours de la Libération, 33405 Talence, France \\ surname. name@ims-bordeaux. fr
}

\begin{abstract}
The topic of Performance Measurement and Management has been investigated for more than twenty five years leading to more than thirty five methods around the world, developed either by researchers or more pragmatically by practitioners, in order to define and implement indicators. Most of them are more oriented for the definition and few for the implementation. Other are simply a list of recommended PI's. Several studies have been done to compare some of these methods and to explain the reasons of PI's implementation failures. The objectives of this paper is to go deeper in detail in the comparison and in a second time to define a generic framework that could help to detect what should contain a generic method for Performance Indicator System definition and implementation and what is the knowledge that must be included in this kind of method to be more efficient.
\end{abstract}

Keywords: Performance measurement, Framework, GERAM.

\section{Introduction}

The topic of Performance Measurement and Management has been investigated for more than twenty five years leading to more than thirty five methods around the world, developed either by researchers or more pragmatically by practitioners, in order to define and implement indicators. Most of these methods are dedicated to the Performance Indicator (PI) definition and few of them are for the implementation. All these methods have been developed independently based on system theory, production management theory or accounting methods, according to the background of developers. Among all these methods, more or less used and well known, one can cite the famous ones or the most used or disseminated around the world as Balanced Score Card [1], the Performance Prism [2], ECOGRAI [3], IPMS [4], Medori [5] or DPMS [6].

Several studies were performed to compare methods for PMS from several points of views. For instance, [7] classifies these methods according to three categories: financial, goal centred and behavioural, concluding that each one has its own advantages to obtain a consistent PIS. In [8] seventeen definitions of what a Business Process Measurement System is, are analysed through methods available or theoretical articles. This analysis was made on the main features of a PIS, on its role in an organisation and on the process of use it. The main conclusions are that a PIS must be 
multi-dimensional (financial and non-financial), must include strategic objectives, performance targets and supporting infrastructure. [9] uses systematic review to analyse few methods in order to detect why some of them are more useful to manage organisations through measure, i.e. to obtain an efficient PIS. The most important aspect is that a method for PIS must help to the definition of detailed action plans extracted from measures, to measure progress, to have a vision (a perspective as a strategic map) and to have cause and effect relationships (links between indicators).

In conclusion of these studies, it is noticeable that several recurrent points exist in each method such as a methodological approach to build the PIS, some basic concepts to define performance indicators as objectives, action means, etc. However, each of them has also some lacks in order to obtain an efficient PIS, easy to build and easy to use for decision makers. So, the conclusion is that each method, even the most famous ones, can be improved based on qualities of other ones.

So, The objective of this paper is to go further not only in the comparison of methods but also in the definition of a generic framework that could help to define what should contain a method to define and implement Performance Indicator System. The objective is not to define a new method by itself but to build the base for the future combination of methods to collect, and to manage in order to use at the best the enterprise knowledge required to build an efficient PIS.

So, in a first part, the paper will explain the difference between a method and a framework and why it is obvious to start from GERAM framework to perform this work. In a second part, a complementary analysis will be presented, highlighting what are the concepts and the components required for a method for PIS design and implementation. In a third time the paper will present GERAM framework developed by IFAP/IFIP taskforce. In a fourth time, GERAM is adapted to the domain of PIS and the base for the framework for PIS methods is presented. Then, perspectives for future works are proposed.

\section{A Framework Dedicated to PIS Methods}

\subsection{Why and What Is a Framework}

As mentioned previously, the few analyses conducted to compare PIS methods show that even if all these methods were based on theoretical or practical points of view, none are complete and none can ensure to collect the whole required knowledge to obtain an efficient PIS for a single enterprise or a supply chain. The objective of this work is to "set in order" the various existing methods. Indeed, the goal of the framework is to answer to the large diversity of objectives pursued by all PIS methodologies, and then to federate them.

A lot of experiences, led in the domain of modelling for Information Systems with Unified Modelling Language [10] or in the domain of enterprise modelling with Unified Enterprise Modelling Language[11], have shown the necessity to work at a generic level of modelling instead of the level of methods in order to obtain coherent models leading to implement efficient systems. This generic level may be constituted of meta-model (such as for UEML), frameworks, etc. This is why it was decided to develop a framework, a kind of meta-model or meta-structure which will help to 
define the required content of a method for PIS definition and implementation. In our point of view, a method is composed of: a set of concepts called also structural framework in [12] which must be identified in the enterprise in order to build the PIS, some formalisms to represent the PIS at work, and a structured approach, called also procedural framework in [12].

A framework is a generalised architecture composed of elements, recommended in PIS engineering and integration, and thereby sets the standard for the collection of related tools and methods. The objective is to allow any enterprise to more successfully tackle initial PIS design, implement and make it evolve during enterprise operational lifetime. This framework does not impose any particular set of tools or methods, but defines the criteria to be satisfied by any set of selected tools and methods.

The main advantage of the framework is not only to present the elements but also the links between these elements. It is clear that the final objective of this framework is also to allow, in the future, to improve each existing method and to inform the practitioners about what to add to these methods.

In order to define this kind of framework, the authors propose to follow the same approach that was used in the domain of enterprise modelling methods and which led to develop GERAM (Generalised Enterprise Reference Architecture and Methodology). GERAM was first selected for two main reasons. The first one is the genericity of the framework that can be applied for each enterprise modelling method. The second reason is the domain of enterprise modelling (EM) which is very closed to the domain of PIS in the sense that EM is the art of externalising enterprise knowledge that adds value to the enterprise (single, extended or virtual organisation). This knowledge is related to the representation of the structure, the behaviour and the organisation of the enterprise according to different points of views: functional, decisional, business, IT... This knowledge is also required to design and implement a PIS.

The interest of this approach linked to GERAM is to avoid to start from scratch when analysing the methods and to define relevant criteria for comparison being specific enough to keep only those interesting for PIS methods. The interest to work at a generic level is to have a global view on the requirements for a PIS without being polluted by a specific context or by a specific function of the enterprise which led to consider only one kind of PI or one decision level. Moreover, the modules are not defined independently in the sense that they are linked together, each one being required to go from user specifications to the implemented Performance Indicator System. For instance, concepts need an architecture to put them in coherence, the architecture need an engineering method to be implemented to define the PIS, which requires a software tool to be used and updated.

\subsection{Methodology of Work}

The methodology used to develop the framework is presented figure 1 .

After a state of the art of existing PIS methods, as exhaustive as possible, a state of the art of existing framework was performed, and GERAM was chosen due to reasons mentioned previously. Then, the various methods were "disassembled" and compared, and commonalities were identified. These commonalities were compared with GERAM modules exposed hereafter. Then, a first version of the framework for PIS methods was elaborated. 


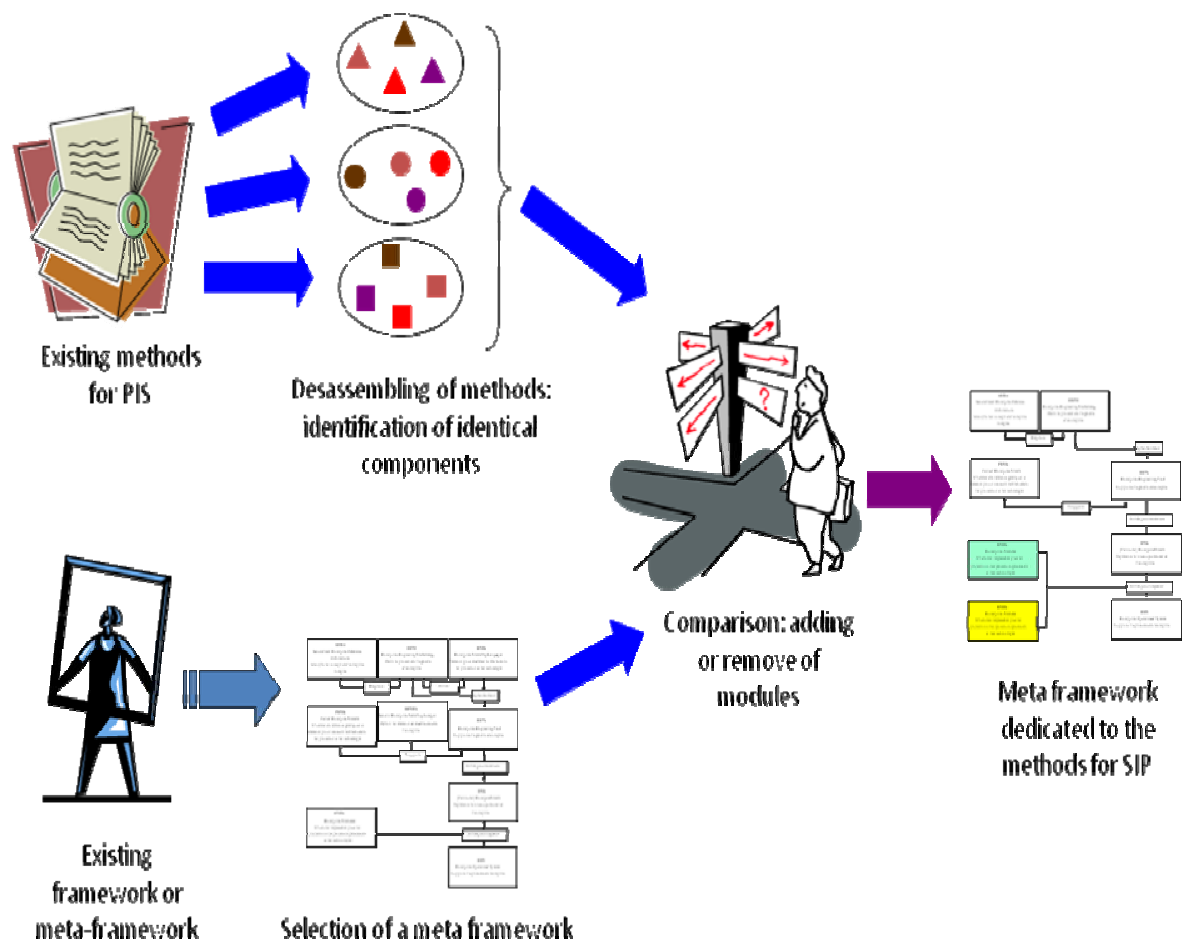

Fig. 1. The methodology of work

\section{The GERAM Framework}

GERAM (Generalised Enterprise Reference Architecture and Methodology) framework was developed by the IFIP-IFAC Task Force on Architectures for Enterprise Integration [13]. This generalised architecture was developed from the evaluation of three existing enterprise integration architectures (CIMOSA, GRAI and PERA) and concluded that each of them had something to offer. To do so, a generalised architecture is required. It defines a tool-kit for designing and maintaining enterprises for their entire life-history and it has the potential for application to all types of enterprises. The set of components identified in GERAM is briefly described in the following:

-GERA (Generic Enterprise Reference Architecture) defines generic concepts recommended for use in enterprise engineering and integration projects. They can be categorised as: human, process and technology oriented concepts.

-EEMs (Enterprise Engineering Methodologies) describe the processes of enterprise engineering and integration in the form of a process model or structured procedure with detailed instructions.

-EMLs (Enterprise Modelling Languages) define the generic modelling constructs adapted to the need of people creating and using enterprise models.

-GEMCs (Generic Enterprise Modelling Concepts) define and formalise the most generic concepts of enterprise modelling which may be defined in 
various way: natural language (glossaries), meta-model, ontological theories defining the meaning (semantics) of modelling languages.

-PEMs (Partial Enterprise Models) are reusable, paradigmatic, typical models which capture characteristics common to many enterprises within or across one or more industrial sectors.

-EETs (Enterprise Engineering Tools) support the processes of enterprise engineering and integration. They should provide for analysis, design and use of enterprises models.

-EMs (Enterprise Models) particular may consist of several models describing various aspects or views of the enterprise.

-EMOs (Enterprise Modules) are physical elements (resources) which can be used in the implementation of the enterprise.

-EOSs (Enterprise Operational System) particular support the operation of a particular enterprise. The implementation is guided by the particular enterprise model which provides the system specifications.

GERAM framework is presented in the figure 2 below:

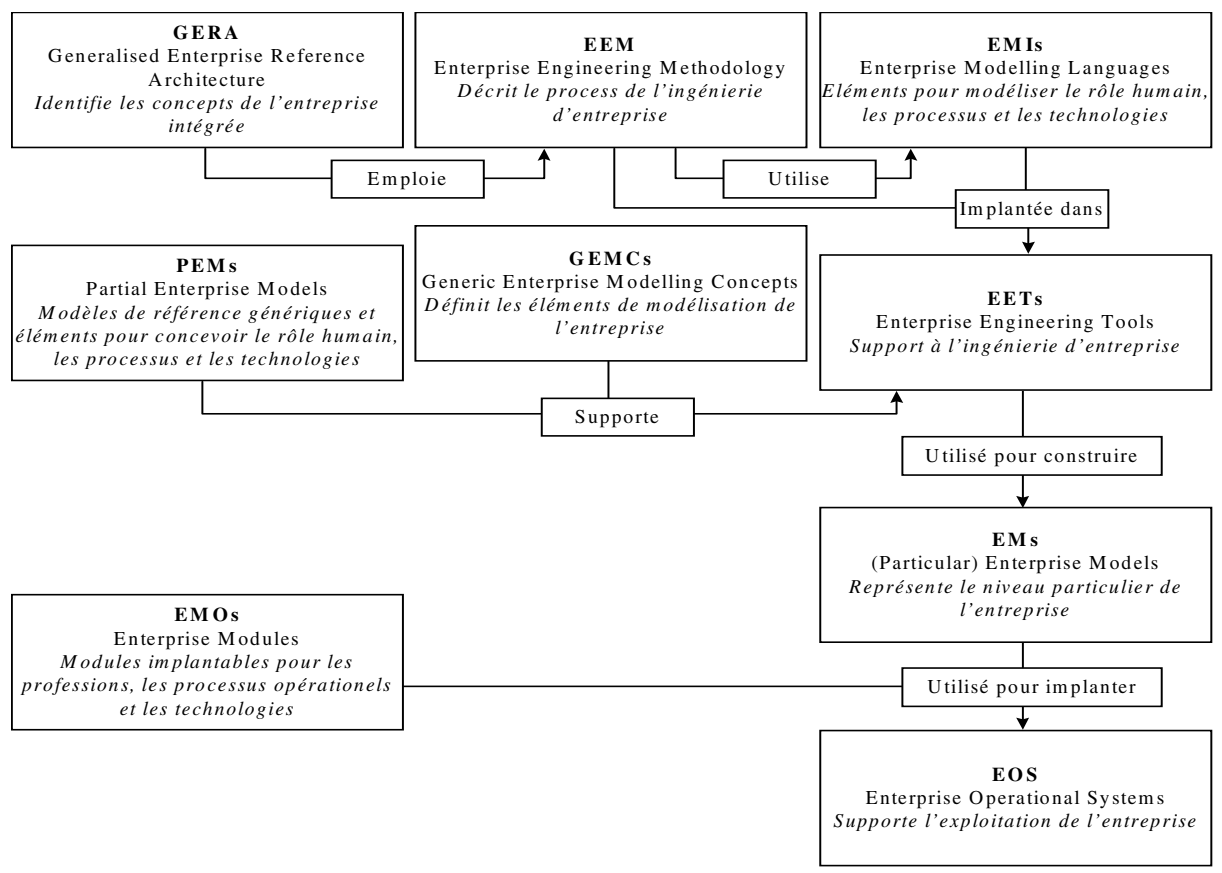

Fig. 2. GERAM Framework

GERAM can be more considered as a set of requirements than as a real practical enterprise modelling method but all the existing methods can consider GERAM to evaluate their completeness. 


\section{The Framework for PIS Methods}

Based on the disassembling of the set of PIS methods and based on the components of GERAM, the following elements are proposed to be included. It means that, in comparison to GERAM, some components are kept because they correspond to PIS methods as for EM methods, other components were removed and then other were added to complete the framework.

\section{GEPISA: GEneral Performance Indicator System Architecture}

GEPISA is a crucial component because it aims to structure the future PIS. This contains guides and rules for designing PIS. This is closed to the structural framework as defined by [12]. GEPISA aims also to describe the way of running for the PIS. GEPISA can contain the different architecture included in the studied methods:

- architectures based on audit methods to seek areas to improve (audit led),

- architectures based on enterprise models (model led),

- architectures based on perspectives (need led).

\section{PISEM: Performance Indicator System Engineering Method}

PISEM describes the process to design and to implement PIS. It is expressed in the form of a structured procedure composed of steps as the procedural framework in [12] or the structured approach of ECOGRAI [3].

\section{GEPISC: GEneral Performance Indicator System Concepts}

GEPISC defines all required concepts to design and implemented PIS as objectives, decision variables, customer...

\section{PPIS: Partial Performance Indicator System}

PPIS concerns the use of reference PIS which enables to save time in the definition of PI's. It provides referential PIS reusable according to a specific context (bank, industry, construction ... sectors). It is materialised for instance by a list of PI's as in [13]. The interest of PPIS is also to allow benchmarking as in ENAPS European Project.

\section{PISET: Performance Indicator System Engineering Tool}

PISET concerns the setting up of a technological infrastructure in order to process various aspects such as data collection, coherence, aggregation and consolidation of data, development and presentation of indicators etc...EIS, OLAP, ROLAP, datawarehouse, datamart, datamining, ETL, etc... belong to PISET. The use of decisional computing technology is essential for the implementation of PIS.

\section{COPIS: Conceptual Performance Indicator System}

COPIS consists is PI's description at the conceptual level, i.e. using description tools as specification sheets, maps... At this stage, the PIS is not concrete nor implemented.

\section{OPIS: Operational Performance Indicator System}

OPIS consists in the PIS at the operational level. In this case, the PIS is implemented and composed of customised/parameterised PISET for the particular enterprise.

It is also possible to define other modules that GERAM does not propose. For instance, based on the literature and on the practice of PIS implementation, it seems 
necessary to have a module dedicated to the choice and implementation of PISET. This module would aim to define the steps and tools for the parameterisation and implementation of PISET to obtain PIS at the operational level.

\section{Conclusions and Perspectives}

The comparison of methods led to the conclusions that each of them has advantages and points to improve, either in the definition or in the implementation of PIS. It was then necessary to determine the required elements of such methods to be efficient.

The interest of a framework is to work at a meta level of comparison and then to avoid to start from scratch when analysing the methods, to define relevant criteria for comparison but to be enough specific to keep only those interesting for PIS methods. The modules are linked together, each one being required to start from user specifications towards the implemented Performance Indicator System. In conclusion, the authors will insist on the necessity to continue the survey of existing methods to define and implement performance indicator systems and the detection of required generic modules.

\section{References}

[1] Kaplan, R.S., Norton, D.P.: The Balanced Scorecard. Harvard Business School Press, Boston (1996)

[2] Neely, A., Adams, C., Kennerley, M.: The performance Prism. The scorecard for measuring and managing Business Success, p. 394. Prentice Hall, Englewood Cliffs (2002)

[3] Ducq, Y., Vallespir, B.: Definition and aggregation of a Performance Measurement System in three Aeronautical workshops using the ECOGRAI Method. International Journal of Production Planning and Control 16(2), 163-177 (2005)

[4] Bititci, U.S., Carrie, A.S., Mcdevitt, L.: Integrated Performance Measurement System: a development guide. International Journal of Operations \& Production Management 17(5-6), 522-534 (1997)

[5] Medori, D.: The development and implementation of an integrated performance measurement framework. In: Proceedings of Performance Measurement-Theory and Practice: International Conference of European Operations Management Association, June 27-29, pp. 313-318 (1998)

[6] Ghalayini, A.M., Noble, J.S., Crowe, T.J.: An integrated dynamic performance measurement system for improving manufacturing competitiveness. International Journal of Production Economics 48, 207-225 (1997)

[7] Kihn, L.A.: Comparing performance measurement approaches. In: PMA Conference, Edinburgh (July 2004)

[8] Franco, M., Maar, B., Martinez, V., Gray, D., Adams, C., Micheli, P., Bourne, M., Kennerley, M., Mason, S., Neely, A.: Towards a definition of a business performance measurement system

[9] Franco, M., Bourne, M.: An examination of the literature relating to issues affecting how companies manage through measures. In: Proceedings of the 3rd International IFIP Workshop on Performance Measurement, Bergamo, June 19-20, 2003, PMA Conference, Edinburgh (July 2004) 
[10] OMG,Object Management Group. Unified Modeling Language Specification, Version 1.5, formal/03-03-0 (2003)

[11] Supply-Chain Operations Reference-model, Overview of SCOR Version 8.0, SupplyChain Council, Inc. (2006), http: / / www . supply-chain . org

[12] Berio, G.: Requirements analysis: initial core constructs and architecture, UEML Thematic Network, Contract no: IST 2001 - 34229, Work Package 3 Deliverable 3.1 (May 2003)

[13] Folan, P., Jagdev, H., Browne, J.: Providing for Inter-Organisational Performance Measurement: Challenges and Concepts. In: Proceedings of the 4th International IFIP Workshop on Performance Measurement "implementation of performance measurement systems for supply chains", Bordeaux, June 27-28 (2005)

[14] GERAM - GERAM: Generalised Enterprise Reference Architecture and Methodology, Version 1.6.1, IFIP-IFAC Task Force on Architectures for Enterprise Integration (March 1999) 